\title{
Time-domain outdoor acoustic simulation of a real-life area using land cover classification identified by airborne hyperspectral imagery
}

\author{
Takuya Oshima ${ }^{1, *}$, Yasuhiro Hiraguri ${ }^{2, \dagger}$ and Kazuma Hoshi ${ }^{3, \dagger}$ \\ ${ }^{1}$ Department of Civil Engineering and Architecture, Faculty of Engineering, Niigata University, \\ 8050 Ikarashi-Ninocho, Nishi-ku, Niigata, 950-2181 Japan \\ ${ }^{2}$ Department of Civil Engineering and Architecture, Tokuyama College of Technology, \\ Gakuendai, Shunan, 745-8585 Japan \\ ${ }^{3}$ Department of Architecture and Living Design, Junior College, Nihon University, \\ 7-24-1 Narashinodai, Funabashi, 274-8501 Japan
}

(Received 25 February 2013, Accepted for publication 21 April 2013)

Keywords: Outdoor acoustics, Land cover classification, Ground acoustic characteristics, Finite-difference time-domain simulations, Airborne hyperspectral imagery PACS number: 43.28.En, 42.68.Wt [doi:10.1250/ast.34.364]

\section{Introduction}

One of the authors has presented a linearized Euler simulation technique for the outdoor sound propagation problem over a real-life urban area using a geometry reconstructed on the basis of digital geographic information [1]. In the technique, the boundary surfaces (the ground and the buildings) were assumed to be perfectly reflecting owing to the lack of an identification technique of the acoustic absorption characteristics of such surfaces from geographic information. In order to solve the identification problem, the authors presented a classification technique of using airborne hyperspectral imagery [2] for land cover types in a real-life area. By this technique, the land area corresponding to each pixel of a hyperspectral image is classified as one of the five ground types defined by ASJ RTN-Model 2008 [3].

In the present study, the identified surface characteristics are applied to a finite-difference time-domain outdoor acoustic simulation in a real-life area. The simulation employs a porous-medium model for the solution of acoustic absorption by the surfaces. A validation of the porous-medium model through comparisons with exact solutions is given. The results are compared with those obtained assuming perfectly reflecting surfaces.

\section{Numerical technique}

Finite-difference time-domain technique developed by Salomons [4] that can deal with acoustic propagation in porous media is employed. The governing equations are

$$
\begin{aligned}
& \frac{\partial \boldsymbol{u}}{\partial t}=-\frac{\Omega}{c_{\mathrm{s}} \rho_{0}} \nabla p-\sigma \frac{\Omega}{c_{\mathrm{s}} \rho_{0}} \boldsymbol{u}, \\
& \frac{\partial p}{\partial t}=-\frac{\rho_{0} c_{0}^{2}}{\Omega} \nabla \cdot \boldsymbol{u}
\end{aligned}
$$

where $\boldsymbol{u}$ is the particle velocity vector $[\mathrm{m} / \mathrm{s}], p$ is the acoustic pressure $[\mathrm{Pa}], c_{0}$ is the speed of sound in air $[\mathrm{m} / \mathrm{s}]$ and is $343.7[\mathrm{~m} / \mathrm{s}], \rho_{0}$ is the air density $\left[\mathrm{kg} / \mathrm{m}^{3}\right]$ and is 1.205

\footnotetext{
*e-mail: oshima@eng.niigata-u.ac.jp

†e-mail: hiraguri@tokuyama.ac.jp

‡e-mail: hoshi@arch.jcn.nihon-u.ac.jp
}

$\left[\mathrm{kg} / \mathrm{m}^{3}\right], \sigma$ is the flow resistivity $\left[\mathrm{kPa} \cdot \mathrm{s} / \mathrm{m}^{2}\right], \Omega$ is the porosity [-], and $c_{\mathrm{s}}$ is the structure constant [-]. In air, the values of $\sigma=0, \Omega=1$ and $c_{\mathrm{s}}=1$ are given.

\section{Sound propagation over a porous surface}

\subsection{Numerical configuration}

As a validation of the implementation of the numerical technique, a sound propagation problem over a porous surface, as shown in Fig. 1(a), is solved. The $x$ and $z$ axes are taken to be horizontal and vertical, respectively. The ranges of the computational domain are $0 \leq x \leq 50[\mathrm{~m}]$ and $-1 \leq z \leq 5.25[\mathrm{~m}]$. The regions below and above $z=0$ are filled with a porous medium and air, respectively. The $x$ directional domain boundaries $(x=0$ and $x=50 \mathrm{~m})$ are rigid. Three types of porous media with the flow resistivities $\sigma=$ $100,1,000$, and $10,000\left[\mathrm{kPa} \cdot \mathrm{s} / \mathrm{m}^{2}\right]$ with $\Omega=0.3$ and $c_{\mathrm{s}}=3$ are tested.

A point source is located at the position $\left(x_{\mathrm{s}}, z_{\mathrm{s}}\right)=$ $(0,0.5)[\mathrm{m}]$. The point source is a Gaussian initial pressure pulse with a full width at half maximum of $0.3125 \mathrm{~m}$. Four receivers are located at $x_{\mathrm{r}}=5,10,20$, and $40[\mathrm{~m}]$ with $z_{\mathrm{r}}=1.5[\mathrm{~m}]$. The grid spacing is $0.03125 \mathrm{~m}$ for the $x$ and $z$ directions. The time integration step is $5 \times 10^{-5} \mathrm{~s}$. The end time of the simulations is $0.15 \mathrm{~s}$ so that the simulations stop just before the propagating wavefront reaches the right boundary of the domain $(x=50 \mathrm{~m})$. The upper domain boundary has a perfectly matched layer (PML) [5] of 20 grid thickness.

The results are compared with an exact solution given by Salomons [4]:

$$
\begin{aligned}
p\left(x_{\mathrm{r}}, z_{\mathrm{r}}, \omega\right)= & j \pi\left\{H_{0}^{(1)}\left(k R_{1}\right)+H_{0}^{(1)}\left(k R_{2}\right)\right\} \\
& -2 \frac{j \pi k}{Z} \int_{0}^{\infty} \exp \left(-\frac{q k}{Z}\right) \\
& \cdot H_{0}^{(1)}\left(k \sqrt{{x_{\mathrm{r}}^{2}}^{2}\left(z_{\mathrm{r}}+z_{\mathrm{s}}+j q\right)^{2}}\right) d q, \\
R_{1}= & \sqrt{x_{\mathrm{r}}^{2}+\left(z_{\mathrm{r}}-z_{\mathrm{s}}\right)^{2}}
\end{aligned}
$$




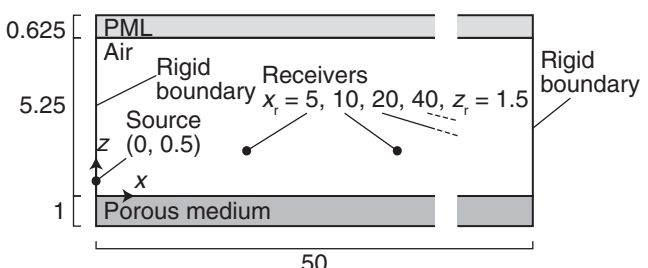

a) Porous medium

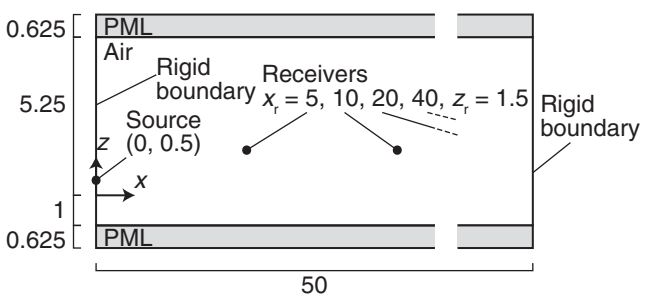

b) Half-space free field

Dimensions: $[\mathrm{m}]$

Fig. 1 Geometries of the ground reflection problem.

$$
\begin{aligned}
R_{2} & =\sqrt{x_{\mathrm{r}}^{2}+\left(z_{\mathrm{r}}+z_{\mathrm{s}}\right)^{2}}, \\
Z & =\sqrt{\frac{q_{c}}{\Omega}}, \\
q_{c} & =\frac{c_{\mathrm{s}}}{\Omega}+j \frac{\sigma}{\rho_{0} \omega}, \\
k & =\frac{\omega}{c_{0}},
\end{aligned}
$$

where $p\left(x_{\mathrm{r}}, z_{\mathrm{r}}, \omega\right)$ is the sound pressure at the receiver position $\left(x_{\mathrm{r}}, z_{\mathrm{r}}\right)$ and angular velocity $\omega, j=\sqrt{-1}$, and $H_{0}^{(1)}$ is the Hankel function of the first kind and order zero.

For comparison, a half-space free-field problem, as shown in Fig. 1(b), is also solved. In the free-field case, the porous medium in Fig. 1(a) is replaced with air. Also, another PML of 20 grid thickness is added at the lower boundary of the computational domain.

\subsection{Results}

Figure 2 shows the numerical and exact solutions of the sound pressure levels relative to those of the half-space freefield cases. In all cases, the numerical and exact solutions agree well in the frequency range up to $500 \mathrm{~Hz}$. In particular, for each case of $\sigma=100,1,000$, and $10,000\left[\mathrm{kPa} \cdot \mathrm{s} / \mathrm{m}^{2}\right]$ with $x_{\mathrm{r}}=5[\mathrm{~m}]$, a deep minimum near a frequency of $600 \mathrm{~Hz}$, owing to destructive interference between a direct wave and a wave reflected at the surface of the porous medium, the numerical solution agrees well with the exact solution.

\section{Solution of a real-life problem}

\subsection{Topographical and numerical configurations}

The subject real-life area shown in Fig. 3 is the test area used in a land cover classification study by Hiraguri et al. [2]. In the classification study, the land is classified into the ground categories defined by ASJ RTN-Model 2008 [3]. Since the ASJ model only provides the effective flow resistivity corresponding to each category, the porosity and the structure constant are adopted from a category with the nearest flow resistivity in another reference [6], as shown in Table 1 (case A).

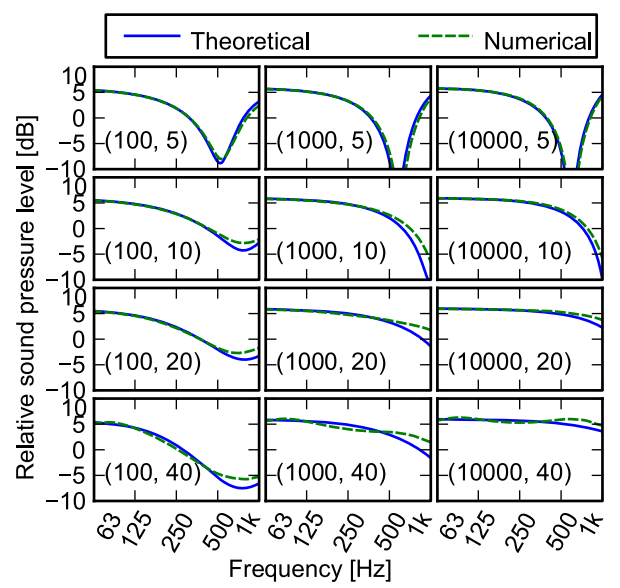

Fig. 2 Theoretical and numerical relative sound pressure levels. Parenthesized numbers denote $\sigma$ $\left[\mathrm{kPa} \cdot \mathrm{s} / \mathrm{m}^{2}\right]$ and $x_{\mathrm{r}}[\mathrm{m}]$.

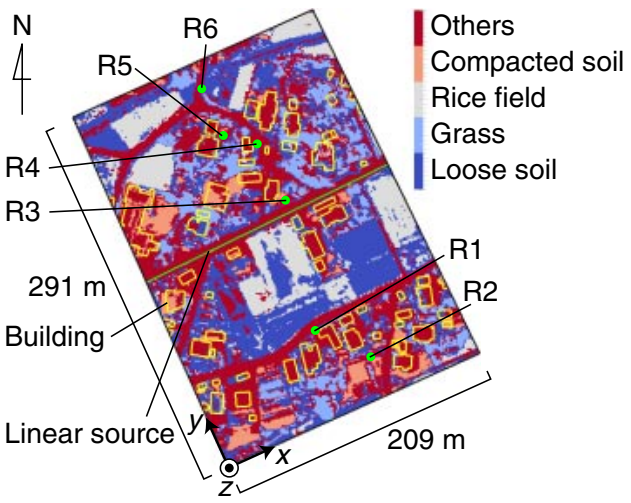

Fig. 3 Subject area.

Table 1 Ground characteristics. The values of $\sigma$ are in $\mathrm{kPa} \cdot \mathrm{s} / \mathrm{m}^{2}$.

\begin{tabular}{ccccc}
\hline & Loose soil & Grass/Rice field & Compacted soil & Others \\
\hline$\sigma$ & 75 & 300 & 1,250 & 20,000 \\
$\Omega$ & 0.4 & 0.5 & 0.3 & 0.1 \\
$c_{\mathrm{s}}$ & 2.25 & 1.69 & 1.96 & 3.24 \\
\hline
\end{tabular}

The terrain and building geometries are reconstructed by the technique developed in a former study [7] using a digital surface model [8] and a building outline dataset [9]. The height of the computational domain is $50 \mathrm{~m}$ (including the height of the ground portion of about $8 \mathrm{~m}$ ). The grid spacing is $0.125 \mathrm{~m}$. With the given grid spacing, the number of grid points is $1,680 \times 2,320 \times 400$ (for the $x \times y \times z$ directions shown in Fig. 3). The lateral and upper domain boundaries are additionally enclosed by a PML of 20 grid thickness. The source is a coherent linear Gaussian pulse with a full width at half maximum of $1.25 \mathrm{~m}$ and an amplitude of $1 \mathrm{~Pa}$ placed on a road, assuming a road traffic noise source, as shown in Fig. 3. The receivers are located at R1-R6 in Fig. 3 at a height of $1.5 \mathrm{~m}$ above the ground. The time integration step is $2 \times$ $10^{-4} \mathrm{~s}$. The end time is $2 \mathrm{~s}$. 


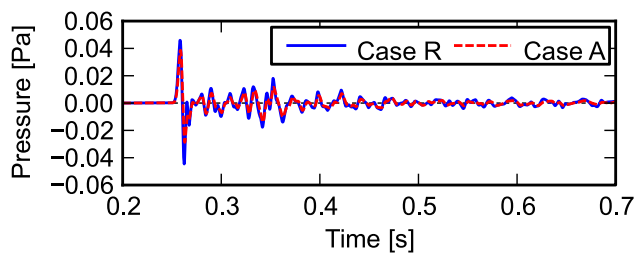

Fig. 4 Pressure waveform obtained at R1.

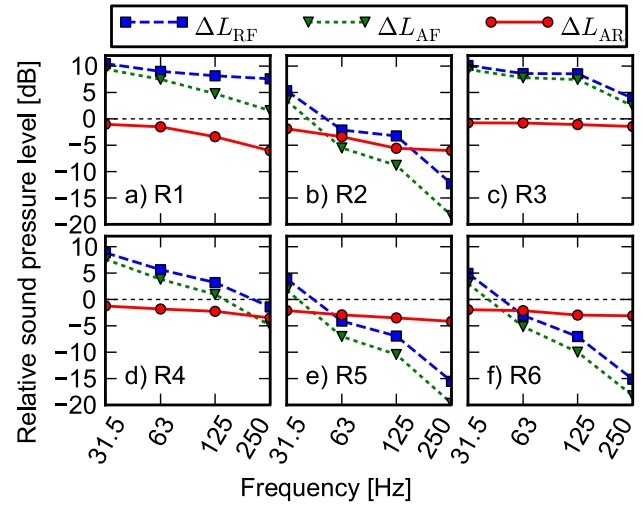

Fig. 5 Octave-band relative sound pressure levels obtained at R1-R6.

For comparison, a case with the same geometry but with rigid surfaces (case R) and a free-field case with the same domain size but with an additional PML at the lower domain boundary (case F) are also solved.

\subsection{Results}

The acoustic pressure waveforms of cases $\mathrm{R}$ and $\mathrm{A}$ at receiver R1 are shown in Fig. 4. In case A, where ground absorption is taken into account, the amplitude is smaller than that in case $\mathrm{R}$.

Figure 5 shows octave-band sound pressure levels for case $\mathrm{R}$ relative to those for case $\mathrm{F}\left(\Delta L_{\mathrm{RF}}\right)$, case $\mathrm{A}$ relative to case $\mathrm{F}\left(\Delta L_{\mathrm{AF}}\right)$ and case $\mathrm{A}$ relative to case $\mathrm{R}\left(\Delta L_{\mathrm{AR}}\right)$. At receiver $\mathrm{R} 1$, where sound propagates over a rice field without being shielded by buildings, $\Delta L_{\mathrm{RF}}$ is positive owing to reflections by the ground and by the buildings behind the receiver. However, $6 \mathrm{~dB}$ of attenuation is observed for $\Delta L_{\mathrm{AF}}$ with regard to $\Delta L_{\mathrm{RF}}$ at the same receiver for a frequency of $250 \mathrm{~Hz}$. The difference demonstrates the effects of the porous property of the surfaces. At receiver R2, which is located behind a building, both $\Delta L_{\mathrm{RF}}$ and $\Delta L_{\mathrm{AF}}$ attenuate with increasing frequency owing to shielding by the building, with additional ground absorption in the case of $\Delta L_{\mathrm{AF}}$. For receivers R3-R5, both $\Delta L_{\mathrm{RF}}$ and $\Delta L_{\mathrm{AF}}$ exhibit attenuation by buildings and by the ground surface by amounts corresponding to the distances between the source and the receivers. However, at receiver R6, which is located in an open area behind a building, no substantial difference is observed with respect to R5.

\section{Conclusions}

In the present study, identified surface characteristics are applied to a finite-difference time-domain outdoor acoustic simulation in a real-life area. The implementation of the porous-medium model employed for the simulation is validated through comparisons with the exact solutions. The numerical results agreed well with the exact solutions. The solutions of the real-life problem are compared with those for the same geometry but assuming perfectly reflecting surfaces. The maximum difference of $6 \mathrm{~dB}$ supports the validity of the present methodology.

The upper bound of the octave bands of $250 \mathrm{~Hz}$ presented in the study, which originates from the limitation in computational resources, does not cover the full spectral content of road traffic noise. However, propulsion noises from road vehicles are known to have dominant spectral contents in the $63 \mathrm{~Hz}$ and $125 \mathrm{~Hz}$ bands [10]. For simulations of such noise, the scale of the simulations presented in the study is applicable in its current state. For more generic road traffic noise prediction including rolling noises that have broader spectra of more than $1 \mathrm{kHz}$, simulations of larger scale should be required. Such larger scale simulations are the subject of future work.

\section{Acknowledgements}

This work is financially supported by JSPS Grant-in-Aid for Scientific Research (B) 23360255. The computational resources are provided by the Joint Usage/Research Center for Interdisciplinary Large-Scale Information Infrastructures under project number 12-NA18.

\section{References}

[1] T. Oshima and M. Imano, "Unified automatic mesh generation of urban terrain for linearized Euler simulations using digital geographic information," Proc. Internoise 2011 (Osaka), No. 429662, 9 pp. (2011).

[2] Y. Hiraguri, T. Oshima and K. Hoshi, "Case study of land cover classification estimation using hyperspectral imaging data for outdoor acoustic simulations," Proc. Inter-Noise 2012 (New York), 8 pp. (2012).

[3] K. Yamamoto, "Road traffic noise prediction model 'ASJ RTN-Model 2008': Report of the research committee on road traffic noise," Acoust. Sci. \& Tech., 31, 2-55 (2010).

[4] E. M. Salomons, "Eulerian time-domain model for sound propagation over a finite-impedance ground surface. Comparison with frequency-domain models," Acta Acust. United Acust., 88, 483-492 (2002).

[5] Q. Qui and T. L. Geers, "Evaluation of the perfectly matched layer for computational acoustics," J. Comput. Phys., 139, 166-183 (1998).

[6] D. K. Wilson, V. E. Ostashev, S. L. Collier, N. P. Symons, D. F. Aldridge and D. H. Marlin, "Time-domain calculations of sound interactions with outdoor ground surfaces," Appl. Acoust., 68, 173-200 (2007).

[7] T. Oshima, Y. Hiraguri and M. Imano, "Geometry and mesh generations for linearized Euler outdoor acoustic simulations using digital geographic information," Tech. Rep. Noise Vib. Acoust. Soc. Jpn., N-2012-10, 8 pp. (2012) (in Japanese).

[8] Kokusai Kogyo Co., Ltd., "RAMS-e Surface level 1," http://www.ramse3d.com/laser/lib/index.html (accessed 2 Apr. 2012).

[9] Geospatial Information Authority of Japan, "Fundamental geospatial data (Accuracy level 2500)," http://www.gsi.go.jp/ kiban/index.html (accessed 24 Feb. 2013).

[10] B. Peeters and G. v. Blokland, The Noise Emission Model for European Road Traffic (Deliverable 11 of the IMAGINE project, 2007), pp. 17-22. 